\title{
Placa oclusal associada às terapias auxiliares para o controle da sintomatologia dolorosa
} do paciente bruxômano

Occlusal splint associated with auxiliary therapies to control the painful symptomatology of the bruxist patient

Férula oclusal asociada a terapias auxiliares para el control de la sintomatología dolorosa del paciente bruxista

Recebido: 13/10/2021 | Revisado: 20/10/2021 | Aceito: 25/10/2021 | Publicado: 28/10/2021

\author{
Maria Eduarda Silveira Guilherme de Oliveira \\ ORCID: https://orcid.org/0000-0002-8405-246X \\ Centro Universitário das Faculdades Metropolitanas Unidas, Brasil \\ E-mail: eduardasilveiragui@gmail.com \\ Silvia Linard Marcelino Friggi \\ ORCID: https://orcid.org/0000-0003-0022-7662 \\ Centro Universitário das Faculdades Metropolitanas Unidas, Brasil \\ E-mail: silvia.linard@hotmail.com \\ Caleb Shitsuka \\ ORCID: https://orcid.org/0000-0002-9813-0457 \\ Centro Universitário das Faculdades Metropolitanas Unidas, Brasil \\ E-mail: caleb.shitsuka@fmu.br
}

\begin{abstract}
Resumo
Objetivos: Através de uma revisão de literatura, fazer um comparativo entre a placa oclusal com terapias coadjuvantes como a acupuntura, fisioterapia, toxina botulínica e laserterapia para o tratamento da parafunção do bruxismo. Métodos: O estudo utilizou literaturas das bases de dados Scielo, Pubmed, Biblioteca virtual em saúde e Ebsco. Foram escolhidos artigos em português e inglês e com data de publicação com menos de 11 anos. Revisão de literatura: O bruxismo é um hábito parafuncional decorrente da contração involuntária dos músculos da mastigação, especialmente os masseteres e temporais. O tratamento mais indicado é a placa oclusal por ser menos invasiva e apresenta uma maior quantidade de estudos provando sua eficiência. Atualmente terapias auxiliares associada a placa oclusal ou isolada tem mostrado resultados efetivos na redução dos sinais e sintomas causados pelo bruxismo. Conclusão: O estudo concluí que a terapia tradicional é efetiva, mas quando associada a outras terapias tem melhores resultados.
\end{abstract}

Palavras-chave: Bruxismo; Tratamento; Placa oclusal; Toxina botulínica; Acupuntura.

\begin{abstract}
Objectives: Through a literature review, make a comparison between occlusal splint and auxiliary therapies such as acupuncture, physiotherapy, botulinum toxin and laser therapy for the treatment of bruxism parafunction. Methods: The study used literature from the Scielo, Pubmed, Virtual Health Library and Ebsco databases. Articles in Portuguese and English and with publication date under 11 years were chosen. Literature review: Bruxism is a parafunctional habit resulting from the involuntary contraction of the mastication muscles, especially the masseters and temporalis. The most indicated treatment is the occlusal splint as it is less invasive and has a larger number of studies proving its efficiency. Currently, auxiliary therapies associated with occlusal or isolated splint have shown effective results in reducing the signs and symptoms caused by bruxism. Conclusion: The study concluded that traditional therapy is effective, but when combined with other therapies, it has better results.
\end{abstract}

Keywords: Bruxism; Treatment; Occlusal splint; Botulinum toxin; Acupuncture.

\section{Resumen}

Objetivos: Mediante revisión de la literatura, realizar una comparación entre férula oclusal y terapias adyuvantes como acupuntura, fisioterapia, toxina botulínica y terapia con láser para el tratamiento de la parafunción del bruxismo. Métodos: El estudio utilizó literatura de las bases de datos Scielo, Pubmed, Virtual Health Library y Ebsco. Se eligieron artículos en portugués e inglés y con fecha de publicación menor a 11 años. Revisión de la literatura: El bruxismo es un hábito parafuncional resultante de la contracción involuntaria de los músculos de la masticación, especialmente los maseteros y 
temporal. El tratamiento más indicado es la férula oclusal por ser menos invasiva y cuenta con un mayor número de estudios que demuestran su eficacia. Actualmente, las terapias auxiliares asociadas a la férula oclusal o aislada han mostrado resultados efectivos en la reducción de los signos y síntomas provocados por el bruxismo. Conclusión: El estudio concluyó que la terapia tradicional es efectiva, pero cuando se combina con otras terapias, tiene mejores resultados.

Palabras clave: Bruxismo; Tratamiento; Férula oclusal; Toxina botulínica; Acupuntura.

\section{Introdução}

O bruxismo é um hábito parafuncional caracterizado por uma atividade repetitiva da musculatura mastigatória que tem como característica o ranger dos dentes de forma inconsciente ou apertar dos mesmos quando o paciente está acordado (Machado, Machado, Cunali \& Fabbro, 2011). O bruxismo é um distúrbio de movimento ligado ao sono (Gonçalves, Toledo \& Otero, 2010). Os sinais e sintomas mais prevalentes são: dor de cabeça, dor orofacial, desgastes dentários, crepitação na ATM, fadiga dos músculos da mastigação, hipertrofia do masseter e temporal, sensibilidade dentária, trinca dentária, fraturas de restaurações e danos ao periodonto (Férnandez-Nunez, Amghar-Maach \& Gay-Escoda, 2019). Essa parafunção pode causar disfunção temporomandibular (DTM), um problema orofacial que afeta a articulação temporomandibular (ATM), músculos da mastigação e estruturas associadas prejudicando o sistema estomatognático (Nishimori et al., 2014).

Durante algum momento da vida $85 \%$ a $90 \%$ da população relata sofrer de bruxismo. (Candirli et al., 2016; Teixeira \& Sposito, 2013). Os fatores etiológicos estão relacionados com os neurotransmissores do sistema nervoso central e podem ser desencadeados por aspectos psicossociais como depressão, estresse e ansiedade, interferências oclusais, distúrbios do sono, desordens neurológicas e efeito colateral de medicamentos (Gonçalves, Toledo \& Otero, 2010; Férnandez-Nunez, Amghar-Maach \& GayEscoda, 2019). O diagnóstico deve ser minucioso com anamnese e exame clínico detalhado, podendo ser necessário realizar um exame complementar que mapeia o sono, a polissonografia, para que outros distúrbios do sono sejam descartados (Steurer et al., 2018; Bisi et al., 2017).

Por tratar de uma etiologia multifatorial, o tratamento do bruxismo necessita de uma abordagem interdisciplinar com cirurgiões dentistas, psicólogos, psiquiatras e fisioterapeutas (Torres, Campos, Fillipini, Weigert \& Vecchia, 2012). Nesse viés, as placas oclusais por serem mais acessíveis que outras terapias, não invasivas e eficiência comprovada cientificamente, são a primeira escolha de terapia inicial para os pacientes bruxômanos. Ademais, toxina botulínica, acupuntura, laser de baixa intensidade e fisioterapia são terapias coadjuvantes para o tratamento do bruxismo (Steurer et al., 2018).

Mediante ao exposto, o objetivo dessa revisão de literatura é, portanto, apresentar os benefícios da placa miorrelaxante como terapia convencional e expor as terapias coadjuvantes para o tratamento do bruxismo.

\section{Metodologia}

O artigo apresenta um Revisão de Literatura Narrativa, que é um critério que descreve e discute um determinado tema através de um ponto de vista teórico. Foi realizado uma pesquisa quantitativa após a seleção das bases de dados, que por sua vez foram: Scielo, Biblioteca virtual em saúde, Pubmed e Ebsco e livros acadêmicos.

As palavras-chave utilizadas foram: bruxismo, tratamento, placa oclusal, toxina botulínica, acupuntura, fisioterapia e laserterapia. Para o estudo ser selecionado, foi optado que no resumo do artigo tivessem pelo menos a palavra bruxismo e um dos tipos de tratamentos citados nas palavras chaves. Os livros que fizeram parte da revisão de literatura, também deveriam conter o assunto do tema. 
Research, Society and Development, v. 10, n. 14, e131101421751, 2021

(CC BY 4.0) | ISSN 2525-3409 | DOI: http://dx.doi.org/10.33448/rsd-v10i14.21751

Foram escolhidos, então, 9 artigos na base de dados Scielo, 7 na Pubmed, 3 no Ebsco, 4 na Biblioteca virtual em saúde e 2 livros. Sendo todos que abordassem de maneira geral o bruxismo e o tratamento. Incluídos no estudo Casos clínicos randomizados, revisões sistemáticas e pesquisas bibliográficas. Os estudos escolhidos deveriam abordar o tema bruxismo e tratamentos, especificamente placa oclusal, toxina botulínica, laserterapia, acupuntura e fisioterapia aplicada aos pacientes bruxômanos. Deveriam incluir definição, etiologia, fisiopatologia, tratamento e seus receptivos benefícios. Ao final 25 literaturas escolhidas.

Os critérios de inclusão também levaram em conta o tempo de publicação, que no máximo, deveriam ter 11 anos e que fossem escritos em português e inglês. Os critérios de exclusão se aplicaram a artigos com data de publicação maior de 11 anos e publicados em idiomas distintos aos supracitados.

\section{Revisão de Literatura}

O bruxismo é uma patologia oral que tem gerado preocupação entre os dentistas devido aos impactos clínicos sobre o sistema estomatognático. A manifestação dessa condição se dá pelo ranger dos dentes durante a noite, conhecido como bruxismo do sono ou excêntrico e durante o período diurno apertando os dentes, denominado bruxismo em vigília ou cêntrico (Canales, Câmera-Souza, Amaral, Garcia \& Manfredini, 2017).

Aproximadamente 20 milhões de brasileiros sofrem de algum distúrbio ligado ao sono e na maioria o tratamento é realizado por uma equipe multidisciplinar. A polissonografia é um exame complementar que auxilia no diagnóstico do bruxismo, pois descarta a existência de outras patologias que possam estar relacionadas ao adormecimento (Lima, Santos, Filho, Bezerra \& Figueredo, 2020; Bisi et al., 2017).

Os estágios do sono foram divididos em 4 fases de sono não REM (Rapid Eye Movements) e a fase REM. Os estágios I e II não REM são caracterizados por sono leve e são observados nos primeiros minutos do início do adormecimento. Já as fases III e IV não REM são relacionadas com o sono mais profundo e por último a fase REM que tem intensa atividade e metabolismo cerebral acelerado. Um estudo polissonográfico realizado por 15 noites em bruxômanos revelou que os episódios de bruxismo podem acontecer em qualquer fase do sono, principalmente nas fases I e II não REM, dificilmente nas fases III e IV, no estudo não foram observadas ocorrências na fase REM diferente de outras pesquisas que notaram os episódios também nesse estágio (Bisi et al., 2017).

Quando ultrapassam a resistência dos tecidos bucais os sinais e sintomas se tornam nítidos. O bruxômano apresenta características clínicas como dor na ATM, hipertrofia dos masseteres e temporais, dor muscular na face, estalos na ATM pela perda da dimensão vertical de oclusão, desgastes incisais dos dentes, reabsorção óssea, além de dor de cabeça. O dente é o mais prejudicado, a parafunção instalada provoca ocorra perda gradativa de esmalte e dentina, compromete o periodonto e em progresso pode causar perda de elemento dental (Lima, Santos, Filho, Bezerra \& Figueredo, 2020).

Em situação de aparecimento do bruxismo sem causas médicas ou sistêmicas, o distúrbio intitula-se bruxismo primário (Cunali et al., 2012). O bruxismo secundário está relacionado com transtornos psicológicos, morfológicos e neurológicos, incluindo transtorno do sono (Lima, Santos, Filho, Bezerra \& Figueredo, 2020). A presença de alterações emocionais e/ou sistêmicas como álcool, idade, DTMs, estresse e ansiedade podem acarretar uma parafunção (Steurer et al., 2018; Lima, Santos, Filho, Bezerra \& Figueredo, 2020).

A etiologia do bruxismo é multifatorial e o tratamento envolve uma equipe multiprofissional. À vista disso, a psicologia atua em controle do estresse e técnicas de relaxamento e higiene do sono. A fisioterapia, por sua vez, atua com auxílio de aparelhos que causam contrações involuntários e relaxamento dos músculos, diminuindo os espasmos e aumento da circulação sanguínea da região (Lima, Santos, Filho, Bezerra \& Figueredo, 2020). A odontologia oferece o tratamento mais escolhido para o bruxismo, o uso 
de placas oclusais para a proteção dos dentes é a primeira escolha de tratamento por alterar os contatos oclusais de forma não invasiva e reversível (Steurer et al., 2018). Além disso, é por meio da odontologia, que é capaz de restaurar elementos dentais para devolver dimensão vertical, usar laser de baixa potência devido seus efeitos analgésicos e fazer uso da toxina botulínica que apresenta eficiência na diminuição da dor orofacial e frequência dos eventos do bruxismo (Lima, Santos, Filho, Bezerra \& Figueredo, 2020; Catão, Oliveira, Costa \& Carneiro, 2013). A técnica de acupuntura através da estimulação de alguns pontos, pode ocasionar liberação de hormônios analgésicos, como exemplo, as endorfinas (Lima, Santos, Filho, Bezerra \& Figueredo, 2020; Zotelli, Meirelles \& Sousa, 2010).

\section{Placa Oclusal}

O uso da placa oclusal é a escolha mais comum para o tratamento de hábitos parafuncionais e disfunções temporomandibulares. Pode ser encontrada com outros nomes como: tala oclusal, placa miorrelaxante, placa de Michigan ou splint oclusal (Nishimori et al., 2014). Sua indicação demanda uma anamnese rica em detalhes, presença ou não de patologia, bom planejamento e habilidade do cirurgião dentista para escolher o tipo de placa mais adequado. A placa macia confeccionada em silicone não requer ajuste oclusal e por isso necessita de um tempo menor de cadeira. Atualmente estudos, como do Candirli et al. (2016), revelam que a eficiência é baixa em comparação a placa oclusal rígida.

A placa oclusal rígida, confeccionada em resina acrílica, é mais efetiva para o tratamento do bruxismo (Steurer et al., 2018; Lima, Santos, Filho, Bezerra \& Figueredo, 2020). É produzida em laboratório e deve abranger toda a superfície oclusal e incisal dos dentes. Pode ser desenvolvida tanto para maxila e mandíbula, mas para o arco superior apresenta maior estabilidade e conforto. A posição de escolha para sua confecção é em relação cêntrica e pode fazer uso de articulador. Após sua confecção, deve apresentar contatos bilaterais simultâneos e em guia anterior e lateralidade os dentes posteriores devem desocluir (Nishimori et al., 2014; Steurer et al., 2018).

A placa oclusal atua como proteção dos elementos dentários, visto que a resina acrílica tem resistência menor ao desgaste que o dente, dessa forma protegendo-os dos movimentos involuntários parafuncionais. Elas são capazes de diminuir os mecanismos que desestabilizam o sistema estomatognático, mediante a promoção de equilíbrio entre a ATM e os músculos da mastigação, o que ocasiona uma melhor posição fisiológica (Steurer et al., 2018).

As placas oclusais são conservadoras, não invasivas, custo acessível e fácil de serem confeccionadas, sendo, como já destacado, a primeira escolha de tratamento para o bruxismo (Steurer et al., 2018). Elas possibilitam uma oclusão próxima ao ideal por proporcionar uma melhor relação entre o disco articular e a cabeça da mandíbula, protege os bruxômanos dos desgastes dentais, evitando dor articular e sensibilidade dental. O uso da placa também é eficiente para pacientes bruxistas e portadores de DTMs por descomprimir a articulação temporomandibular e promover uma distribuição de carga para diferentes regiões de tecidos articulares e dentários (Nishimori et al., 2014).

Os hábitos parafuncionais causam danos aos elementos dentais e ao periodonto, necessitando, então, de intervenção para que não progridam e cause consequências mais severas. Essas atividades anormais resultam contração muscular prolongada. $\mathrm{O}$ mecanismo de ação da placa oclusal é atuar como redutora da atividade muscular aumentando a dimensão vertical de oclusal reduzindo a carga da ATM, proteção dos elementos dentais e das estruturas de suporte da força parafuncional (Steurer et al., 2018).

Os hábitos parafuncionais são capazes de desestabilizar o aparelho estomatognático e ocasionar DTMs. Os sintomas e sinais mais comuns da disfunção temporomandibular são: tensão da musculatura mastigatória, dificuldade de fazer os movimentos mandibulares, estalos e crepitação articular, dor na região de ATM, sendo este último o principal motivo dos pacientes buscarem 
Research, Society and Development, v. 10, n. 14, e131101421751, 2021

(CC BY 4.0) | ISSN 2525-3409 | DOI: http://dx.doi.org/10.33448/rsd-v10i14.21751

tratamento. O uso de placas oclusais para o tratamento de DTMs promove diminuição de dor e desconfortos na ATM. Além disso, a placa aumenta a dimensão vertical e afasta o contato entres os dentes, o que gera diminuição do esforço muscular e consequentemente cause menos dores articulares (Steurer et al., 2018).

É necessário que todo paciente que faz o uso de placas oclusais tenha acompanhamento periódico com um dentista e que sejam colaborativos ao aderir ao tratamento. É válido destacar que essa terapia não é a cura do bruxismo, mas proporciona redução da sintomatologia e aumenta a qualidade de vida do bruxômanos. Outro ponto é que não é o único tratamento para hábitos parafuncionais. $\mathrm{O}$ fracasso do tratamento pode acontecer por negligenciar as sessões de estabilização que são necessárias pós a instalação da placa e pela falta de colaboração dos pacientes (Steurer et al., 2018; Nishimori et al., 2014).

\section{Toxina botulínica}

Os tratamentos para bruxismo têm como foco principal promover relaxamento muscular, e várias terapias conseguiram esse resultado, incluindo a toxina botulínica (Canales, Câmera-Souza, Amaral, Garcia \& Manfredini, 2017). Alguns pacientes não conseguem aderir aos tratamentos convencionais, por isso a terapia com aplicação de Toxina botulínica tipo A (BTX-A) se torna hábil já que não necessita da colaboração diária do paciente (Teixeira \& Sposito, 2013). Atualmente, esse método ganhou espaço em diversos estudos, que apontam que o BTX-A é eficaz para controlar os músculos mastigatórios com movimentos involuntários e o bruxismo secundário (Shim et al., 2014).

Pesquisas recentes apontam que o bruxismo é causado por níveis altos de atividade muscular nos músculos ligados a mandíbula, de modo que a recorrência da contratura muscular gera inflamação e hipóxia muscular local ocasionando dor orofacial (Jadhao et al., 2017). A BTX-A é uma neurotoxina que tem capacidade quando aplicada no músculo esquelético de inibir a liberação da acetilcolina nos terminais nervosos e motores, causando, desse modo, relaxamento muscular (Férnandez-Nunez, Amghar-Maach \& Gay-Escoda, 2019; Teixeira \& Sposito, 2013; Canales, Câmera-Souza, Amaral, Garcia \& Manfredini, 2017; (Dall'Magro et al., 2015).

Segundo o Comitê Educacional WE Move a dose recomendada de injeção de toxina botulínica é de 40 UI por músculo tratado, mas os valores podem variar de 8 a 100 UI para os masseteres e de 0 a 40 UI para os temporais A justificativa para maior concentração injetada nos masseteres é que são músculos mais volumosos dos que os temporais, necessitando, assim, de uma quantidade maior para gerar efeito. A ação máxima é observada entre o $7^{\circ}$ e $14^{\circ}$ dias após a aplicação e os efeitos podem durar até 6 meses, mas a média de duração é entre 3 a 4 meses (Teixeira \& Sposito, 2013; Canales, Câmera-Souza, Amaral, Garcia \& Manfredini, 2017).

Aplicações de BTX-A em músculos masseteres e temporais não diminui os episódios de bruxismo, mas reduz a intensidade de contração dos músculos mastigatórios provocando diminuição da sintomatologia dolorosa (Shim et al., 2014). O estudo realizado por Jadhao et al. (2017), recrutou 24 pacientes com diagnóstico de bruxismo para comprovar a eficácia da toxina botulínica. Os pacientes foram divididos de forma randomizada em 3 grupos: o primeiro foi tratado com a BTX-A, o segundo recebeu injeções de placebo com solução salina e o terceiro foi o grupo controle, no qual nenhuma aplicação de injeção ocorreu.

Existem algumas contradições sobre a aplicação da BTX. Os efeitos adversos mais leves apontam hematomas, dor no local da aplicação, fraqueza muscular, disfagia, boca seca e dor de cabeça (Férnandez-Nunez, Amghar-Maach \& Gay-Escoda, 2019; Jadhao et al., 2017). Além disso, houve um crescimento no número de pesquisas que relatam que a aplicação da toxina intramuscular pode gerar atrofia do músculo e como consequência, induzir reabsorção óssea. O osso mandibular é estimulado pela força gravitacional dos músculos mastigatórios, no momento em que estes diminuem a sua função, puxam o osso com menos força, 
podendo, por conseguinte, ocasionar um possível quadro de osteopenia por desuso (Hong \& Kang, 2020; Chang, Bergeron, Chen \& Chen, 2011).

Hong e Kang (2020) realizaram uma pesquisa com 39 mulheres jovens e 38 mulheres pós-menopausa para comparar a qualidade da cortical óssea antes e depois da aplicação de BTX-A. Outro estudo importante para avaliar espessura da cortical óssea, espessura mandibular e o volume mandibular total pós terapia de Botox foi realizado por Chang, Bergoron, Chen e Chen (2011) com 10 mulheres entre 20 e 40 anos de idade. Foram feitos 20 exames de tomografia computorizadas nas pacientes, 10 antes e 10 após 3 meses do tratamento com aplicação de doses terapêuticas de BTX-A.

\section{Fisioterapia}

O bruxismo é um fator de risco para o começo de uma DTMe seus efeitos geram alterações funcionais e estruturais da ATM e dos músculos mastigatórios, que, quando afetados causam danos as estruturas associadas (Steurer et al., 2018; Cunali et al., 2012). Para tratar dessas disfunções o dentista é alvo de primeira escolha para o tratamento, porém, atualmente o bruxismo é visto como algo mais complexo e quando instalado seus efeitos podem atingir a musculatura do ombro e pescoço, acarretando desarmonias posturais e/ou esqueléticas necessitando, por isso, da intervenção de fisioterapeutas (Teixeira \& Sposito, 2013; Torres, Campos, Fillipini, Weigert \& Vecchia, 2012).

A fisioterapia é útil como terapia coadjuvante no tratamento de dor associada a articulação temporomandibular e os músculos ligados a ela (Steurer et al., 2018). Suas técnicas contam com massagem manual, exercícios, terapia de liberação posicional, neuro estimulação elétrica transcutânea (tens), alongamentos e ultrassom para contribuir minimizando as sequelas adversas do bruxismo, bem como as DTMs e proporcionar uma melhora na qualidade de vida dos portadores dessas disfunções (Torres, Campos, Fillipini, Weigert \& Vecchia, 2012; Segar, Nunes, Pivetta \& Lima, 2012).

A dor orofacial é o sintoma que mais faz com que as pessoas busquem por tratamento e seu aparecimento na maioria das vezes é decorrente da fadiga e espasmos musculares causados pela intensa hiperatividade dos músculos da mastigação (Torres, Campos, Fillipini, Weigert \& Vecchia, 2012). A massagem manual contribui reduzindo a sintomatologia dolorosa, por intermédio de técnicas de manipulação da pele, exercícios e alongamentos que melhoram a elasticidade do músculo, induzem a liberação de opióides endógenos que aumentam a circulação sanguínea e estimulam a formação de líquido sinovial que faz a lubrificação da ATM (Lima, Santos, Filho, Bezerra \& Figueiredo, 2020; Segar, Nunes, Pivetta \& Lima, 2012).

O ultrassom melhora significativamente as queixas dos bruxômanos, seu mecanismo de ação funciona por oscilações de calor transmitidas pelo tecido que atingem até $5 \mathrm{~cm}$ de profundidade, causando relaxamento dos músculos, redução da dor crônica e alongamento do tecido mole (Torres, Campos, Fillipini, Weigert \& Vecchia, 2012; Leeuw, 2010). A eletroterapia, especialmente o Tens, quando aplicada na superfície cutânea, age contra a estimulação sensorial dos impulsos dolorosos (Segar, Nunes, Pivetta \& Lima, 2012). Torres, Campos, Fillipini, Weigert e Vecchia (2012) separaram em 2 grupos com 5 pacientes cada: primeiro grupo recebeu tratamento fisioterápico com aplicação de tens, ultrassom e alongamentos e no segundo apenas odontológico com utilização de placa oclusal e medicamento, o objetivo era comprovar a efetividade das técnicas usadas na fisioterapia.

O desequilíbrio do sistema estomatognático pode causar, como mencionado, consequências posturais. A fisioterapia evita que os músculos do pescoço, ombros, bem como os mastigatórios e linguais desempenhem suas funções de forma errada, evitando, assim, cirurgias, por meio do reposicionamento mandibular, melhora da postura, reduzindo inflamação e sobrecarga da ATM (Torres, Campos, Fillipini, Weigert \& Vecchia, 2012; Segar, Nunes, Pivetta \& Lima, 2012, Leeuw, 2010). Com exceção de momentos de fala, mastigação e deglutição, a mandíbula deve ser posicionada posterior à maxila e permanecer relaxada com os arcos desocluídos. 
Os exercícios ajudam aos pacientes a evitar atividades danosas as ATMs, ensinando os alongamentos e relaxamentos dos músculos (Leeuw, 2010).

\section{Acupuntura}

A acupuntura é um método milenar da medicina chinesa conhecida por sua aplicabilidade no controle da dor. O sistema nervoso periférico é estimulado em pontos estratégicos do corpo denominados acupontos por meio de agulhas descartáveis e muito finas que agem ativando a liberação de neurotransmissores capazes de promover bem-estar. A literatura sugere que os acupontos designados para tratar o bruxismo são E6 (Jiache) e TA17 (Yifeng) localizados respectivamente acima ao ângulo da mandíbula e atrás do lóbulo da orelha e para tratamento de tensão muscular facial, os pontos VB20 localizado na região posteroinferior do crânio na borda inferior do osso occipital medialmente ao processo mastoide e VB21 encontrado entre a região do pescoço e do ombro, na altura do acrômio (Steurer et al., 2018; Zotelli, Meirelles \& Sousa, 2010; Santos, Recco, Mota, Holanda \& Junior, 2017; Donatelli, 2011).

Na pele e nos músculos existem fibras nervosas que quando estimuladas, enviam para medula espinhal impulsos nervosos que por sua vez ativam o mesencéfalo, hipotálamo e a hipófase. Em resposta, há liberação de endorfina, serotonina, noradrenalina, dinorfina, neurotransmissores responsáveis por impedir a sintomatologia dolorosa, benéficos no controle do estresse e ansiedade. Além desses neurotransmissores, também ocorre liberação de histaminas, íons de potássio e prostaglandinas que diminuem o limiar excitatório dos músculos. A acupuntura não trata apenas o local de punção, mas possui o objetivo de equilibrar todo o corpo (Zotelli, Meirelles \& Sousa, 2010; Santos, Recco, Mota, Holanda \& Junior, 2017).

No bruxismo, a dor oriunda dessa disfunção gera impactos negativos sociais, emocionais, bem como a falta de concentração e estresse (Segar, Nunes, Pivetta \& Lima, 2012). A acupuntura apresenta uma boa alternativa de tratamento, devido à sua eficácia no aumento de amplitude de movimento mandibular, relaxamento muscular e uma analgesia de princípio lenta, conquanto duradoura. Os neurotransmissores catecolaminas estão relacionados com estresse e ansiedade e são desencadeadores do bruxismo. A aplicação de agulhas em acupontos estratégicos proporciona diminuição de catecolaminas resultando na melhora do bruxismo. Além de analgesia, a acupuntura é benéfica por melhorar a circulação sanguínea da região, além de atenuar espasmos e inflamação (Zotelli, Meirelles \& Sousa, 2010; Santos, Recco, Mota, Holanda \& Junior, 2017).

\section{Laserterapia}

O sistema estomatognático é composto por várias estruturas, e cada uma possui sua determinada limitação. Hábitos parafuncionais causam hiperatividade muscular, baixa circulação sanguínea e resultam na aglomeração de metabólitos nas células musculares, gerando espasmos, cansaço e dor orofacial (Catão, Oliveira, Costa \& Carneiro, 2013; Assis, Soares \& Victor, 2012). Nessa perspectiva, O uso do laser de baixa intensidade (LBI) vem se tornando uma opção de terapia auxiliar para o tratamento dessas desordens dos músculos esqueléticos faciais (Leeuw, 2010).

A utilização do laser do tipo infravermelho é uma opção válida por ser de custo acessível e não invasiva. Dentre os efeitos dessa terapia, destacam-se ações anti-inflamatórias, analgésicas, melhora da modulação celular e, consequentemente, na cicatrização dos tecidos (Catão, Oliveira, Costa \& Carneiro, 2013; Assis, Soares \& Victor, 2012). Melchior, Brochini e Silva (2017) realizaram uma pesquisa para comparar o uso da placa oclusal associada ao Laser de baixa intensidade no tratamento de disfunções temporomandibulares ocasionadas por hábitos parafuncionais. A pesquisa foi realizada em dois grupos: o primeiro composto por 15 sujeitos, que foram tratados apenas com placa oclusal e o segundo formado por 5 pacientes, tratados com placa associada a aplicações 
Research, Society and Development, v. 10, n. 14, e131101421751, 2021

(CC BY 4.0) | ISSN 2525-3409 | DOI: http://dx.doi.org/10.33448/rsd-v10i14.21751

de laserterapia 2 vezes por semana. O Laser infravermelho foi aplicado nos músculos masseteres e temporais com comprimento de onda $780 \mathrm{~nm}$, potência fixa de $70 \mathrm{mw}$ e doses de $105 \mathrm{~J} / \mathrm{cm}^{2}$ durante 60 segundos por ponto dolorido.

\section{Discussão}

O bruxismo é um hábito parafuncional ligada ao sono que se manifesta pelo contato não funcional dos dentes (Machado, Machado, Cunali \& Fabbro, 2011). O sono é vital para manter o bem-estar dos seres vivos e muitos estudos apontam que o hábito parafuncional ocorre nos momentos de despertar, precisamente nas fases de sono não REM I e II que são caracterizados por sono leve. O estudo de Bisi et al. (2017) mostra que o bruxismo não pode ser observado na fase REM, na qual verifica-se grande atividade neural. Em contrapartida Rugh et al. (1998) apud Bisi et al. (2017), discorrem que é possível que aconteça, e que os casos que ocorrem na fase REM apresentam maior sintomatologia dolorosa.

A etiologia não é exata, mas acredita-se está relacionada a fatores emocionais, neurológicos, inflamatórios, traumas e má higiene do sono (Nishimori et al., 2014). Não existe cura certa para o bruxismo, sendo assim, o papel dos dentistas, psicólogos, médicos e fisioterapeutas se concentram no controle da sintomatologia dolorosa e prevenção de danos às estruturas associadas. $\mathrm{O}$ tratamento é indicado quando a parafunção começa a gerar a perda de estrutura dental, perda óssea, hipertrofia massetérica e do temporal, disfunções de ATM, fratura de restauração e dor de cabeça (Dall’Magro et al., 2015).

Em 2011, Machado, Machado, Cunali e Fabbro ao analisarem a eficácia das placas certificaram-se de que não tratam a gênese do bruxismo, mas são benéficas para o controle dos sinais e sintomas. As placas macias são utilizadas pela facilidade de serem confeccionadas e por demandarem um tempo menor de cadeira, porém atualmente pesquisas apontam efeitos adverso no uso delas. O estudo realizado por Candirli et al. (2016) revelam que as placas macias aumentam a dor e registros polissonográfico da atividade do bruxômanos em relação às placas rígidas. As placas oclusais que não podem ser ajustadas, ou seja, as macias, não são indicadas para tratamento de disfunções de ATM. Enfatiza-se que a placa oclusal é eficiente para tratar o bruxismo, mas não pode ser vista como única opção (Torres, Campos, Fillipini, Weigert \& Vecchia, 2012).

A injeção da toxina botulínica não trata a origem do bruxismo, mas apresenta eficiência no controle da sintomatologia reduzindo a intensidade de contração da musculatura (Canales, Câmera-Souza, Amaral, Garcia \& Manfredini, 2017). Jadaho et al. (2017) revelam que o grupo que recebeu injeção de BTX-A houve redução significativa da força oclusal, em comparação entre o grupo placebo que recebeu dose de solução salina, e o grupo controle no qual não foi injetado nenhuma substância. Após 6 meses de tratamento também foi observado tempo menor da ocorrência do aperto. Nos demais grupos não houve mudanças significativas. Shim et al. (2014), em seu estudo realizado em pacientes bruxômanos com aplicações de BTX-A em músculos masseteres e temporais revelou a ação da neurotoxina não diminui os episódios de bruxismo, mas diminui a intensidade de contração dos músculos mastigatórios, ocasionando diminuição da sintomatologia dolorosa.

Shim et al. (2014) afirmam que o BTX-A é igualmente eficaz em comparação ao uso de aparelhos intraorais na redução da sintomatologia dolorosa. A aplicação da toxina botulínica causa menos desconforto que a placa oclusal, incômodo relatado pelos bruxômanos principalmente ao dormir e não depende totalmente da colaboração do paciente para ter sucesso na terapia (Bisi, et al., 2017). Já a intervenção com placa oclusal depende dessa cooperação para obter resultados e necessitam de ajuste semestralmente e além de quando em uso atrapalha a fonação (Nishimori et al., 2014). A desvantagem do BTX-A em relação a placa é que seu efeito tem duração curta de no máximo 6 meses, necessitando de outra aplicação (Teixeira \& Sposito, 2013). 
Research, Society and Development, v. 10, n. 14, e131101421751, 2021

(CC BY 4.0) | ISSN 2525-3409 | DOI: http://dx.doi.org/10.33448/rsd-v10i14.21751

Os benefícios como diminuição da rigidez muscular, dor e ranger de dentes durante o sono podem ser notados pós terapia (Canales, Câmera-Souza, Amaral, Garcia \& Manfredini, 2017). Problemas na eficiência da terapia pode estar relacionado com erro da dosagem, armazenamento do produto inadequado, erro técnico e/ou resistência a BTX-A (Teixeira \& Sposito, 2013).

Em 2013, Teixeira et al. afirmaram que o efeito da toxina botulínica para o tratamento do bruxismo diminui o evento da parafunção pela redução da atividade muscular, mas não consegue apresentar consequência sobre o sistema nervoso central e que seus efeitos colaterais são inexistentes. Tal posicionamento diverge do estudo apresentando por Hong e Kang (2020) com 39 mulheres jovens e 38 mulheres pós-menopausa, que apresentou que a injeção da toxina botulínica influência nos locais de inserções dos músculos mastigatórios ocasionando atrofia muscular e alteração da qualidade da cortical óssea, especialmente nas mulheres pósmenopausa. Em contrapartida, Chang, Bergeron, Chen e Chen (2011), após 3 meses de tratamento, os resultados dos exames revelaram que após aplicação de BTX-A os masseteres perderam 30\% do seu volume, outros músculos com pterigoide medial e lateral e temporal permaneceram estáveis. No estudo, os autores apontaram que não houve alterações ósseas significativas na mandíbula.

Torres, Campos, Fillipini, Weigert e Vecchia (2012) realizaram uma pesquisa com 10 participantes, 5 tratados apenas com técnicas de fisioterapia e os outros 5 com placa oclusal e medicamento. O desfecho dessa pesquisa revelou que os pacientes tratados com fisioterapia obtiveram resultados mais relevantes comparados aos pacientes que foram tratados com placa. São necessários mais estudos para comprovar esse resultado, pois Silva et al. (2007) apud Torres. et al (2012) afirmam que os resultados da placa apareceram após 70 dias de intervenção, diferente da ação fisioterapêutica que tem efeito mais rápido. Uma revisão sistemática realizada no ano de 2006 declara que a terapia manual, juntamente com outras técnicas de fisioterapia podem ser tão eficientes quanto a intervenção de placa oclusal ou placebos para o tratamento do bruxismo. A terapia manual em pontos de gatilhos é eficiente para redução de dor na ATM e para aumentar a amplitude de movimento da mandíbula (Segar, Nunes, Pivetta \& Lima, 2012).

$\mathrm{O}$ uso da acupuntura tem mostrado bons resultados com ações analgésicas, anti-inflamatórias e ansiolíticas, além de revigorar o sistema imunológico (Lima, Santos, Filho, Bezerra \& Figueiredo, 2020). Blasco et al. (2017) apud Santos et al. (2017), aponta que após 7 dias da terapia de acupuntura para tratamento de dor no masseter e temporal, ocorreu melhora da dor pela estimulação de pontos de gatilhos. Os resultados da terapia em comparação aos tratamentos convencionais, têm decorrências semelhantes, mas são necessários mais estudos clínicos para a correta indicação da acupuntura para o bruxismo (Leeuw, 2010).

A utilização do laser como terapia auxiliar vem crescendo e trazendo bons resultados na diminuição da necessidade cirúrgica ou medicamentosa no tratamento de DTMs. Suas vantagens implicam na suavização da dor e no seu potencial reparador tecidual (Catão, Oliveira, Costa \& Carneiro, 2013). O melhor resultado na pesquisa do Melchior, Brochini e Silva (2017) no grupo com placa associada ao laser é explicado pois o LBI tem efeito biomodulador que melhora a flexibilidade muscular. O estudo apontou também redução maior da dor em pacientes com a placa oclusal associada ao laser do que apenas o grupo tratado com placa oclusal, devido a ação analgésica proporcionada por ele. Os resultados obtidos apontam que ambos os tratamentos foram eficientes, com aumento relevante da amplitude de movimentos mandibulares, mas o grupo com aplicações de laser apresentou resultados mais significantes. Apesar do crescimento na utilização do laser para tratar os sinais e sintomas das disfunções temporomandibulares, sua efetividade ainda não foi totalmente compreendida (Steurer et al., 2018). 


\section{Conclusão}

De acordo com a revisão realizada, a intervenção de placa oclusal é eficiente para o tratamento das parafunções, mas que nem em todos os casos elas são eficazes e que não podem ser vistas como a única opção de tratamento, podendo recorrer ao auxílio de outras terapias coadjuvantes.

De maneira sucinta, a toxina botulínica tem vantagem em relação a placa por apresentar redução dolorosa poucos dias pós terapia e não depender da colaboração do paciente. A fisioterapia tem resultados instantâneos pós-intervenção sendo útil para pacientes com dor aguda. $\mathrm{O}$ uso da acupuntura tem resultados semelhantes ao uso da placa além de promover aumento da imunidade corporal, mas tem poucas pesquisas sobre o assunto necessitando de mais estudos. A laserterapia associada a placa tem resultados mais relevantes do que o uso da placa isolada, por promover uma melhor analgesia da região. Fica claro, dessa forma, que as terapias auxiliares são úteis e tem resultados significativos na diminuição do controle da dor causada pelo ranger ou apertar dos dentes, devolvendo qualidade de vida para os bruxômanos.

\section{Referências}

Assis, T. D. O., Soares, M. D. S., \& Victor, M. M. (2012). O uso do laser na reabilitação das desordens temporomandibulares. Fisioterapia em Movimento, 25(2), $453-459$.

Bisi, M. A., Selaimen, C. M. P., Martins, E. A., Pinto, D. V., Butzke, K. W., \& Valente, H. R. (2017). Características polissonográficas em pacientes bruxômanos. Revista da Faculdade de Odontologia, 12(3), 74-78.

Canales, G. T., Câmera-Souza, M. B., Amaral, C. F., Garcia, R. C. M. R., \& Manfredini, D. (2017). Is there enough evidence to use botulinum toxin injections for bruxism management? A systematic literature review. Clinical Oral Investigations, 21(3), 727-734.

Candirli, C., Korkmaz, Y. T., Celikoglu, M., Altintas, S. H., Coskun, U., \& Memis, S. (2016) Dentists' knowledge of occlusal splint therapy for bruxism and temporomandibular joint disorders. Nigerian journal of clinical practice, 19(4), 496-501.

Catão, M. H. C. V., Oliveira. P. S. D., Costa, R. D. O., \& Carneiro, V. S. M. (2013). Avaliação da eficácia do laser de baixa intensidade no tratamento das disfunções têmporo-mandibular: estudo clínico randomizado. Revista CEFAC, 15(6), 1601-1608.

Chang, C. S., Bergeron, L., Yu, C. C., Chen, P. K. T., \& Chen, Y. R. (2011). Mandible changes evaluated by computed tomography following botulinum toxin a injections in square-faced patients. Aesthetic Plastic Surgery, 35(4), 452-455.

Cunali, R. S., Bonotto, D. M. V., Machado. E., Hilgenberg, P. B., Bonotto, D., Farias, A. C. D., \& Cunali, P. A. (2012). Bruximo do sono e disfunções temporomandibulares: revisão sistemática. Revista Dor, 13(4), 360-364.

Dall'Magro, A. K., Santos, R. D., Dall'Magro, E., Fior, B., Matiello, C. N., \& Carli, J. P. D. (2015). Aplicações da toxina botulínica em odontologia. Salusvita, 34(2), 371-382.

Donatelli, S. (2011). Caminhos de Energia: Atlas dos Meridianos e Pontos para Massoterapia e Acupuntura. Roca.

Fernández-Núnez, T., Amghar-Maach, S., \& Gay-Escoda C. (2019). Efficacy of botulinum toxin in the treatment of bruxism: Systematic review. Medicina Oral, Patologia Oral y Cirurgia Bucal, 24(4), 416-424.

Gonçalves, L. P. V., Toledo, O. A. D., \& Otero, S. A. M. (2010). Relação entre bruxismo, fatores oclusais e hábitos bucais. Dental Press Journal of Orthodontics, 15(2), 97-104.

Hong, S. W., \& Kang, J. H. (2020). Decreased mandibular cortical bone quality after botulinum toxin injections in masticatory muscles in female adults. Scientific Reports, 10(1), 1-12.

Jadhao, V. A., Lokhande, N., Habbu, S. G., Sewane, S., Dongare, S., \& Goyal, N. (2017). Efficacy of botulinum toxin in treating myofascial pain and occlusal force characteristics of masticatory muscles in bruxism. Indian Journal Dental Research, 28(5), 493-497.

Leeuw, R. (2010). Dor orofacial: Guia de avaliação, diagnóstico e tratamento (4a ed). Quintessence.

Lima, M. C. G., Santos, A. P. C., Filho, E. O. N., Bezerra, R. L., \& Figueiredo, R. J. A. (2020). A parafuncionalidade do bruxismo: da intervenção terapêutica multiprofissional ao uso da placa miorrelaxante. Brazilian Journal of Health Veriew, 3(4), 8910-8918.

Machado, E., Machado, P., Cunali, P. A., \& Fabbro, C. D. (2011). Bruxismo do sono: possibilidades terapêuticas baseadas em evidências. Dental Press Journal of Orthodontics, 16(2), 58-64. 
Research, Society and Development, v. 10, n. 14, e131101421751, 2021

(CC BY 4.0) | ISSN 2525-3409 | DOI: http://dx.doi.org/10.33448/rsd-v10i14.21751

Melchior, M. D. O., Brochini, A. P. Z., \& Silva, M. A. M. R. (2017). Laserterapia de baixa intensidade associada ao uso de placa oclusal no tratamento de disfunção temporomandibular: estudo clínico controlado. Revista Dor, 18(1), 12-17.

Nishimori, L. E., Martins, J. R., Marson, F. C., Sábio, S., Silva, C. O., \& Corrêa, G. O. (2014) Utilização de placas oclusais em resina acrílica no auxílio do tratamento de DTMS. Revista UNINGÁ Review, 17(1), 59-64.

Santos, J., Recco, P., Mota, G., Holanda, A. V., \& Junior, V. E. S. (2017). Tratamento da dor orofacial através da acupuntura em pacientes com bruxismo: Um estudo de revisão. Revista da Faculdade de Odontologia, 22(1), 96-100.

Segar, G. T. P., Nunes, S. F., Pivetta, H. M. F., \& Lima, F. S. T. (2012). A terapia manual nos sinais e sintomas do bruxismo do sono. Fisioterapia Brasil, 13(6), 463468 .

Shim, Y. J., Lee. M. K., Kato, T., Park, H. U., Heo, K., \& Kim, S. T. (2014). Effects of botulinum toxin on jaw motor events during sleep in sleep bruxism patients: a polysomnographic evaluation. Journal of Clinical Sleep Medicine, 10(3), 291-298.

Steurer, R., Silva, H. V., Linden, M. S. S., Trentin, M. S., Miyagaki, D. C., \& De Carli, J. P. (2018). Uso de placas oclusais como tratamento de alterações no sistema estomatognático. Revista Salusvita, 37(3), 716-729.

Teixeira, S. A. F., \& Sposito, M. M. M. (2013). A utilização de Toxina Onabotulínica A para bruxismo: Revisão de Literatura. Revista Brasileira de Odontologia, 70(2), 202-204.

Torres, F., Campos, L. G., Fillipini, H. F., Weigert, K. L., \& Vecchia, G. F. D. (2012). Efeitos dos tratamentos fisioterapêutico e odontológico em pacientes com disfunção temporomandibular. Fisioterapia em Movimento, 25(1), 117-125.

Zotelli, V. L. R., Meirelles, M. P. M. R., \& Sousa, M. D. L. R. (2010). Uso da acupuntura no manejo da dor em pacientes com alterações na articulação temporomandibular (ATM). Revista de Odontologia UNICID, 22(2), 185-188. 